\title{
Implementation of Ethernet Passive Optical Network (EPON)
}

\author{
Gurpreet Kaur \\ M.Tech Student, Department of Electronics and Communication Engineering, \\ Punjabi University, Patiala-147002 \\ Dr. Manjeet Singh Patterh \\ Professor, Department of Electronics and Communication Engineering, \\ Punjabi university, Patiala-147002
}

\begin{abstract}
An Ethernet Passive Optical Network (EPON) extends the network to a passive optical splitter filter which would combine Time Division Multiplexing and Mode Division Multiplexing methods to realize a large-scale network access. In this proposed research, we have studied the performance of the bi-directional EPON in both downstream and upstream traffic by utilizing bi-directional optical fiber and power splitter. We have proposed to design a hybrid architecture based on EPON using both TDM and MDM.The transmission of both the network MDM-TDM is experimentally demonstrated over 1.8 - $\mathrm{km}$ bidirectional optical fiber with bit error-free performance to validate the concept. This proposed architecture obtains high-speed transmission over a large-scale passive optical network by combining the multiplexing dimensions. The result has been observing that when the number of users and fiber length has been increased, bit error rate is increased as well, but the maximum $Q$ factor and eye height are decreased due to dispersion.
\end{abstract}

Keywords -ETHERNET, BIT ERROR RATE, PASSIVE OPTICAL NETWORK, PONIT-TO-MULTIPOINT

\section{INTRODUCTION}

Optical networks are becoming more powerful and dynamic with a specific end goal to enhance system performance for high bandwidth traffic.The Point-to-Multipoint topology is a typical network architecture for open air wireless networks to interface different areas to one single central area. In a point-to-multipoint wireless Ethernet network, every remote area does not communicate specifically with each other however have a single association towards the focal point of the star network where one or more base station is regularly found.[4]

This technology together with the demand among service suppliers to automate their physical layer has prompted the advancement of in-service signal generating and switching on optical wavelengths. As network usefulness moves to the physical layer, it is characteristic to expect that monitoring capacities will likewise move into the physical layer. This in-service optical monitoring, which is utilized to pick up data concerning the quality and attributes of the optical signal, is frequently referred to as OPM (Optical Performance Monitoring). Optical fiber links permit us to transmit an ultra-stable frequency with no degradation. [3]

The bandwidth demand of the users is increasing gradually. Till now, PON technology covered lesser transmission distance with alesser number of users. [3] Therefore, more efficient multiplexing architectures are required to realize the large-scale transmission with high-speed supporting large number of users. To obtain large-scale passive optical network, the multiplexing dimensions can be combined to get the high-speed transmission. In this paper, we proposed an MDM-TDM-PON architecture that uses low mode crosstalk FMF. We need networks which fulfill this requirement, and the modern passive optical network (PON) are such networks which provide higher transmission speed on large-scale. PON technology can be coupled with MDM (Mode Division Multiplexing) and TDM (Time Division Multiplexing) to enhance the transmission speed in two-dimensional architecture. [1] So, we proposed to design a hybrid architecture based on Ethernet Passive Optical Network (EPON) using both TDM and MDM. 


\section{PON (PASSIVE OPTICAL NETWORK)}

PON (Passive Optical Networks) are beneficial for their larger bandwidth and low operational costs. There are many PON based networks; these are WDM-PON (Wavelength Division Multiplexing), MDM-PON (Mode Division Multiplexing), and GPON (Gigabit PON) that uses TDM technique. In a recent study, EPON (Ethernet PON) are main attention as a low-cost equipment that uses low-cost fiber infrastructure of PONs. [1]

A PON is a point-to-multipoint optical network utilizing passive optical parts, for example, couplers and splitters with no active components.PON could receive a tree, tree-and-branches, ring or bus topology with tree topology being the most widely recognized. A PON could be utilized to implement a fiber-to-the-home (FTTH), fiber-to-the-building (FTTB) or fiber-to-the-curb (FTTC) subscriber access network.

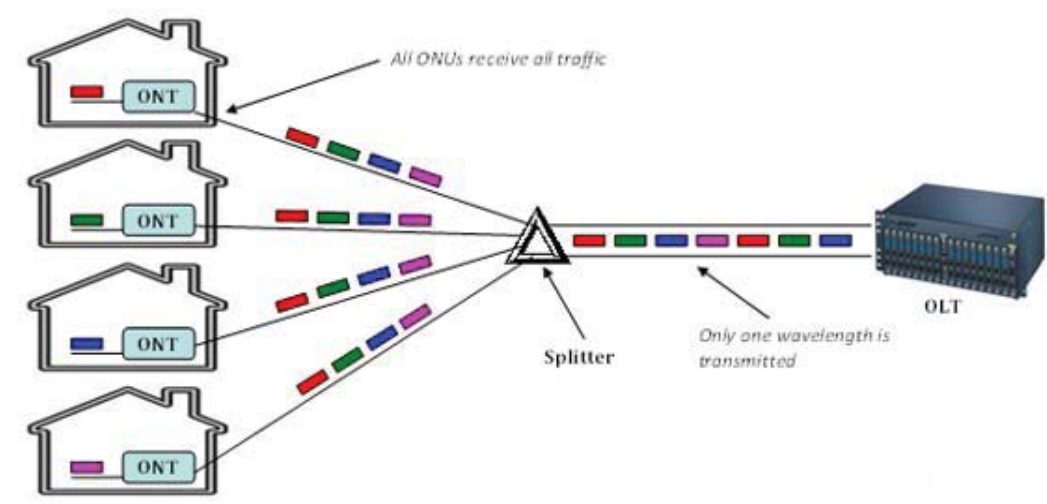

Fig. 1: Passive Optical Network (PON)

In wireless MIMO systems, multipath propagation causes linear distortion, with a delay spread depending mainly on the environment.

\section{DIFFERENT PON TECHNOLOGIES}

EPON cannot be measured either a point-to-point network or shared medium; rather, it is a combination of bothnetworks. It has a connectivity of a point-to-point medium in the upstream direction, and it behaves as a shared medium in the downstream direction. [5]

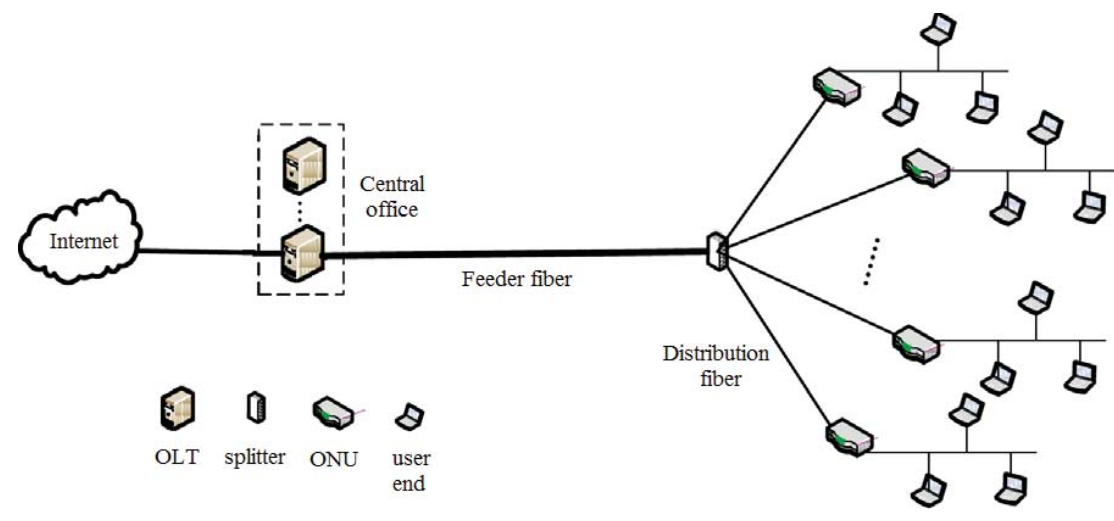

Fig. 2: EPON Network Architecture

Ethernet protocol (EPON) is a packet-based network contact. Therefore, it is optimized for transporting Internet Protocol (IP). Although Internet access is now a major, possibly, amost important service provider offering residential applications require a wide range of services such as data, voice, and video.[6] 


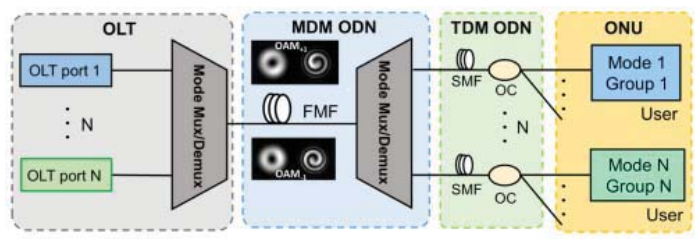

Fig. 3: MDM-TDM Architecture

The OLT(Optical Line Terminal) is like a router orswitch, and the optical network terminal connects to the MAN or WAN, also known as the backbone. Also the network assembling and access functions, OLT also perform the bandwidth assignments, network security, and management patterns as per the client requirements. [6] The ONUis located at the end-user or curb, and it provides optical interfaces which linkedto the OLT and interfaces at users side. [7] The OD (Optical Distributed Network) is mostly composed of one or more passive optical splitters. These splittersconnect the OLT and ONU, which split thedownstream signal from one fiber into several fibers and combine upstream optical signals from multiple fibers into one.

Time-division multiplexing (TDM) is a technique for putting different data streams in a composite signal by isolating the signal into numerous segments, each having a brief span. Every individual data stream is reassembled at the less than a desirable end given the scheduling.

Among the diverse PON technologies, TDM(Time Division Multiplex) based point to multipoint PON innovation norms GPON and EPON have turned into the most broadly conveyed technologies for FTTH and FTTB.

In the TDM-PON technologies, there is a mutual architecture in which a single wavelength is shared amongst subscribers. There is an Optical Network Terminal (ONT) toward the end of every fiber situated in the home or building of the supporter, and a broadcast plan is utilized for downstream data. For the upstream, each ONT has a period slot in which to communicate with the OLT (optical line termination unit) at the central office. [4]

Mode division multiplexing (MDM) explores the longitudinal dimension to increase the transmission capacity in optical fiber communication. It multiplexes different modes. WDM is widely used in the optical fiber to avoid crosstalk problem. Two ways have been launched by $10-\mathrm{m}$ length index multimode optical fiber using conventional gradual-spatial filtering techniques. As crosstalk between the two ways in the baseband is much lower at the exit end of the fiber.Modal and multiplexing seem possible through the short fiber lengths. [1]

WDM-PON includes multiple wavelengths in the fiber to build capacity in the access technology system. While physically it is like TDM based PON technologies, legitimately it is a point to point network. Each ONT at the subscriber has a committed wavelength, and there is no time sharing of bandwidth. The limitation to the bandwidth that the client can get relies on upon what the wavelength can transfer. The splitter in TDM PON systems is supplanted by an arrayed waveguide grating (AWG) which transfers every wavelength to its destination ONT. Dissimilar to TDM PON system, a subscriber gets just the data bound to it. WDM-PON has the guarantee of enhancing the measurements of bandwidth, split ratio, and range over customary PON technologies. With WDM-PON, a virtual PON can be made for every subscriber utilizing multiple waves on a single fiber. [4]

\section{RELATED WORK}

J. Li et al. [1]proposed MDM-TDM-PON (Mode division Multiplexing-time division multiplexing-passive optical network) architecture for the next-generation passive optical network (PON). They demonstrated MDMTDM-PON upstream and downstream transmission experimentally over $2 \mathrm{~km}$ and $1.8 \mathrm{~km}$ with error free transmission. The crosstalk in both methods was very low. So the implemented architecture is promising to construct large monitoring networks. They proposed a new multiplexing technique mode division multiplexing (MDM) within the passive optical network (PON) to realize large- scale access. This technique uses both mode multiplexer/ demultiplexer and few-mode fiber. Hence, it removes the necessity of MIMO (multi Input Multi- 
output).N. Naim et al. [2]implemented monitoring technique for P2MP TDM-EPON. They used L-band ASE source for monitoring the optical signal. They used two FBGs (Fiber Bragg gratings) having different bandwidth, reflectivity, and Bragg wavelength.O. Lopez et al. [3] transmitted untestable frequency to two different users using the single link input. They implemented unique branching network topology. Their architecture enables us to establish an ultra-stable frequency distribution to many users. They also show that the setting up of extraction device, or a simpler one, at the primary link input, can test the accurate functioning of the noise rejection on this core link.S. D. and Ravneet Kaur [4] proposed that PON(passive optical network) was characterized by low cost, high bandwidth, and high reliability. They provided a solution for future access networks by PON through bandwidth capacity and long transmission distance. They described that their offered architecture is suitable for high data rate PON applications like cable TV, Broadband and in optical signal processing.G. F. M. Lima et al. [5] represented a different strategy for stimulation of Optical Time Domain Reflectometry (OTDR) measurement signal. Fiber to the home (FTTH) needs new technologies that would economically and effectively cope with massive fiber plants. S. Kakkar[6] studied some traditional approaches which are replaced by PON( passive optical network). She explained the shortcomings of traditional approaches. She explained the working and applications of EPON( Ethernet passive optical network).Min Zhu [7] explained three technologies in a WDM-PON, including multicast overlay scheme, tunable Fabry-Perot laser diode (FPLD) self-seeding scheme and automatic protection switching scheme.He used WDM optical network as virtual computing environment, to connect distributed resources to support large-scale engineering, scientific and commercial computing applications.T. Muciaccia et al. [8] compared the various existing standards. They thoroughly overviewed the issues in PON. They also proposed future solutions for these problems.N. Mohd Radzi [9] studied on dynamic bandwidth allocation (DBA) which is a major problem in EPON (Ethernet Passive Optical Network). He explained EPON, it'sworking, and applications. He briefly described the classification of the DBA algorithms.D. M. Ruchi Malhotra [10] developed a complete probabilistic study for estimating the packet delay performance of next-generation Passive optical networks (PONs). Sheanalysis also improves the network efficiency and reliability. Shealso demonstrated the identification of network blockages using the analysis. M. Ma et al. [11] they have proposed a novel MAC protocol for the EPON to provide efficient network service to different kinds of optical network units (OUNs) with various bandwidth requirements.K. S. et al. [12]implemented a sample EPON scenario built on the features which are already offered by OPNET along with the IEEE 802.3ah standard definitions. Their implementation comprises all the steps which an Optical Network Unite (ONU) should take to be able to direct traffic towards Optical Line Terminal (OLT) over thehigh-speed upstream optical link.A. Shami et al. [13] proposed a novel hybrid Granting (HG) protocol specifically to recover the performance of services in EPON (Ethernet passive optical network). They proposed dynamic scheduling algorithm which minimizes jitter and packet delay to a large extent for delay and delay-variation sensitivetraffic by assigningbandwidth in a GBR (grant-before-report) fashion.

\section{IMPLEMENTATION OF EPON}

To implement the EPON in simulation technique, we discuss the number of techniques like how the time division multiplexing and mode division multiplexing are varied from each other while performing theoperation in the EPON. As simulation shown in optisystem7.0, how to execute the performance in simulation technique to demonstrate the result in BER (Bit Error Rate) analyzer or Eye diagram.

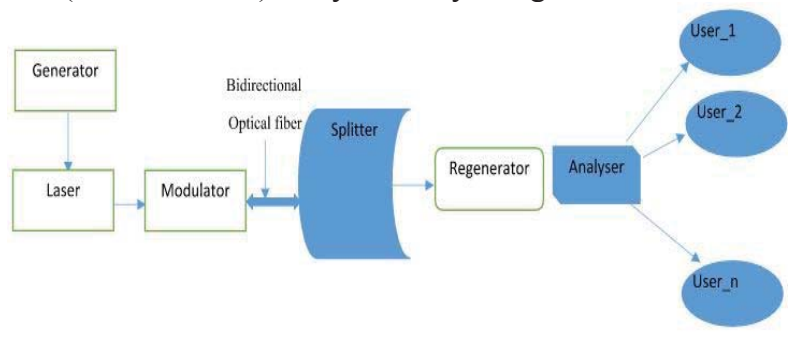

Fig.4: Schematic diagram of EPON simulation

The figure1 indicates that how to send a data packet to the various users. Modulator, control the amplitude of an optical wave came from the continuous laser beam. The ability of light to travel in both direction beside an optical fiber without interfering allows us to use them for bidirectional communication. As regenerator improves 
the transmission distance from where the packet has been travel. BER analyzermeasures the quality of the transmission links to generate the result in the form of graphs. It shows, the quality of data send to the user individually. The result accurately depends on the changing in the fiber length. Min BER and Quality factor indicate that the quality of data sends through Optical Line Terminal to Optical network unit. It sends data to the individual to the user at particular interval of time in the form of packets.

In an implementation, we show the performance of bi-directional opticalfiber through TDM and MDM. In TDM, indicates that the how the optical fiber length travel controlled the data from both directions during the time interval. In MDM, both index fiber is implementing the procedure of measuring the bandwidth that supports the use of laser sources on multimode fiber. These index fibers depend on the spatial coherence.

\section{COMPARISON OF TDM-MDM EPON}

As we discuss earlier the implementation is performed a simulation technique in the optisystem 7.0 , the result shows us the how the quality of data sends to the end-user while using the bi-directional optical length $1.8 \mathrm{~km}$ in two modes. Both the ends of the optical fiber connect with the transmitter and the receiver. How much data sends while we consider time delay approx $0.8 \mathrm{~s}$ or more at the same fiber length.
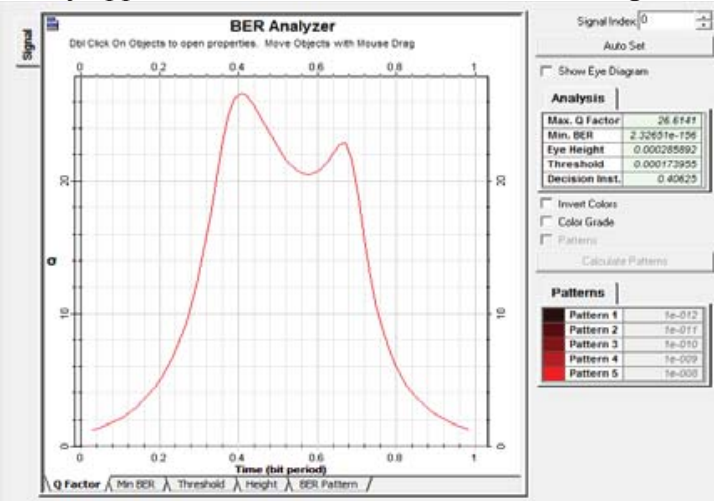

Fig. 5: Time division multiplexing quality factor

The fig.5. depicts the quality factor of data send to the users while performing the time interval on the $1.8 \mathrm{~km}$ optical fiber length.

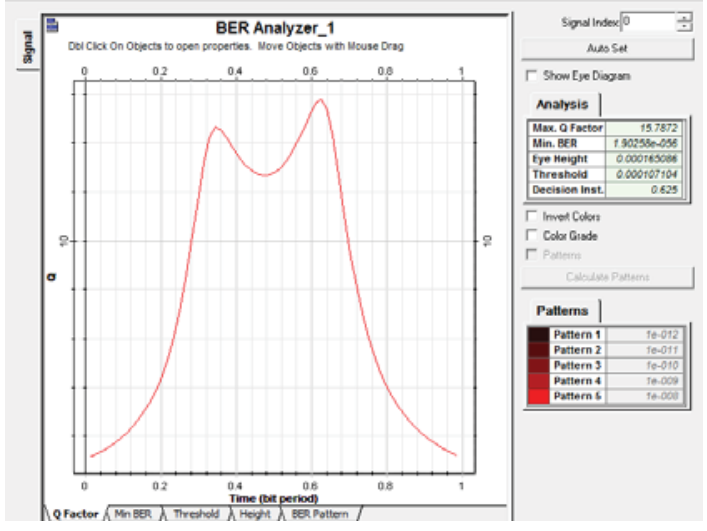

Fig. 6: Mode division multiplexing quality factor

While fig. 6 depicts the quality factor at the same optical length in mode division multiplexing that how the index fiber is measuring the bandwidth that supports the spatial laser.

Both describes that how the result performed in the form of modes and time interval. In how much quality factor data send through packets.

Here the graph shows a comparison between TDM and MDM in EPON. 


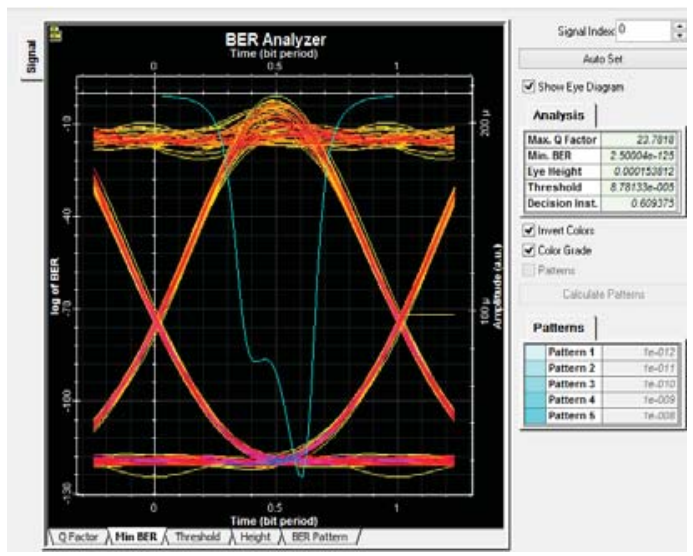

Fig. 7: BER analyzer of TDM

Fig. 7 graph indicates the Min BER value of Time division multiplexing. Min BER value is 2.50004e-125 on the quality factor 23.7 .
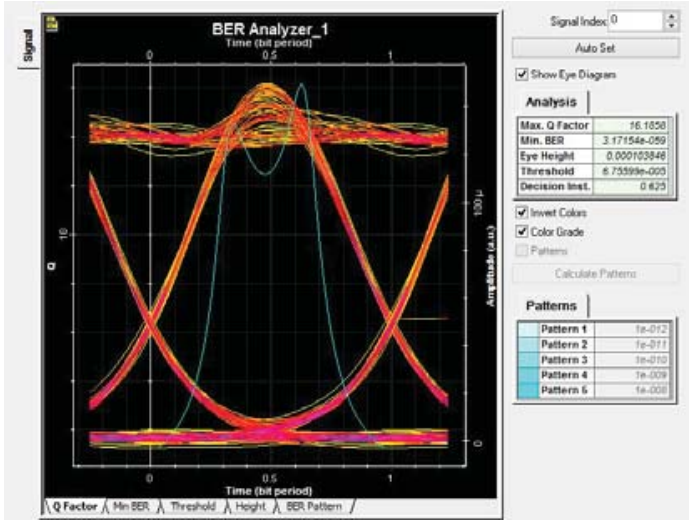

Fig. 8: BER analyzer of MDM

Fig. 8 graph indicates Min BER value of Mode division multiplexing. Min BER 3.17154e-059 and the quality factor 16.1858 .

Both the graphs shows the quality data send to the users while changing the optical fiber length. MDM shows less distortion than TDM because of no time interval while transferring the data through the same length of optical fiber length.

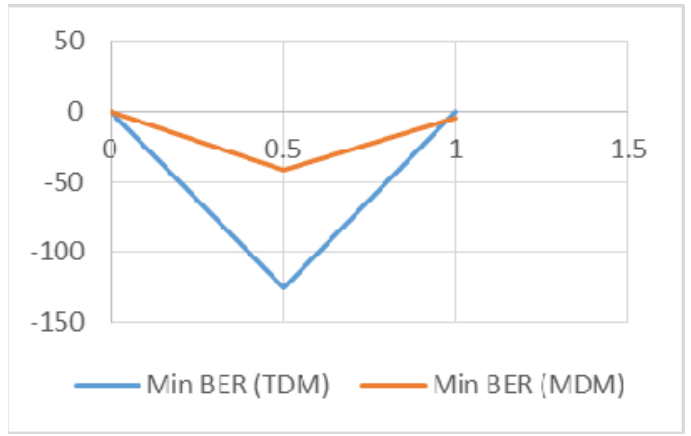

Fig. 9:BER analyzer performance of downstream 


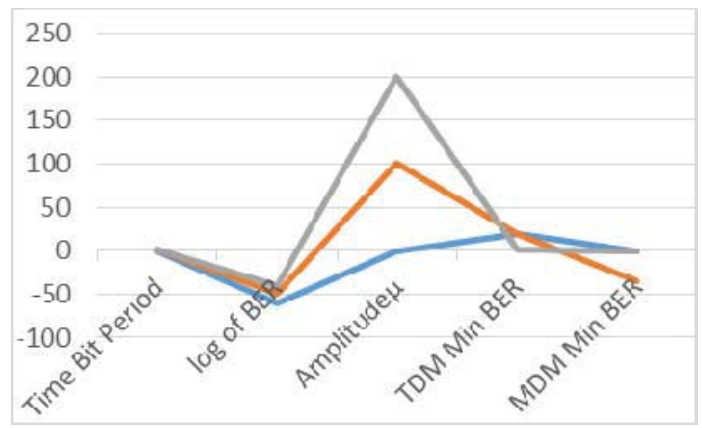

Fig. 10BER analyzer performance of upstream

Analyze the value of TDM and MDM

TABLE1: DOWNSTREAM TDM-MDM EPON PERFORMANCE

\begin{tabular}{|c|c|c|}
\hline FACTORS & $\begin{array}{c}\text { TIME DIVISION } \\
\text { MULTIPLEXING }\end{array}$ & $\begin{array}{c}\text { MODE- DIVISION } \\
\text { MULTIPLEXING }\end{array}$ \\
\hline MAX Q FACTOR & 23.7818 & 15.8066 \\
\hline MIN. BER & $2.50004 \mathrm{e}-125$ & 0.000104116 \\
\hline EYE HEIGHT & 0.000153812 & $7.1633 \mathrm{e}-005$ \\
\hline THRESHOLD & $8.78133 \mathrm{e}-005$ & 0.625 \\
\hline DECISION INST. & 0.609375 & \\
\hline
\end{tabular}

TABLE2: UPSTREAM TDM-MDM EPON PERFORMANCE

\begin{tabular}{|c|c|c|}
\hline FACTORS & $\begin{array}{c}\text { TIME DIVISION } \\
\text { MULTIPLEXING }\end{array}$ & $\begin{array}{c}\text { MODE- DIVISION } \\
\text { MULTIPLEXING }\end{array}$ \\
\hline MAX Q FACTOR & 21.953 & 17.0836 \\
\hline MIN. BER & $3.95212 \mathrm{e}-107$ & $9.83733 \mathrm{e}-066$ \\
\hline EYE HEIGHT & 0.000151122 & 0.000105279 \\
\hline THRESHOLD & $8.91586 \mathrm{e}-005$ & $7.02284 \mathrm{e}-005$ \\
\hline DECISION INST. & 0.609375 & 0.625 \\
\hline
\end{tabular}




\section{GAPS IN LITERATURE}

By conducting the literature survey it has been found that the every monitoring technique has some limitations; i.e. no one is perfect in every case and most of the existing literature has neglected one of the following:

1. The Most of the existing researchers has analysed the system only on the basis of number of users, BER and quality factors were not considered.

2. The monitoring technique for P2MP TDM-EPON implemented but does not reduce the number of equipment.

\section{ANALYTICAL SOLUTION}

In this architecture design, PON hascombined TDM and MDM methods to realize a large-scale network access. We proposed to implement EPON architecture to enhance the transmission speed over large-scale. We set up a downstream transmission of MDM-TDM-PON. PON is characterized by extremely high reliability, low cost, and high bandwidth. PON provides a competent solution for future access networks because of large bandwidth capacity and long transmissiondistance. [2] The utilization of available wavelength is increased by PON and achieves a smooth upgrade under the premise of without disturbance of original users, abusinesswhich improves the scalability of thenetwork.In downstream transmission, signals were modulated by an AWG (arbitrary waveform generator) that splits the light into two beams. This then converted the signal to a different mode and then this signal was transmitted to free space. It has been found that both the downstream MDM and upstream MDM-TDM transmission are suitable to transmit over $1.8 \mathrm{~km}$ few-mode fiber and $20 \mathrm{~km}$ standard single mode fiber. The results of this implementation can be used to create a hybrid architecture of EPON.

\section{CONCLUSION}

In this proposed research, the performance of the bi-directional EPON in both downstream and upstream traffic by utilizing bi-directional optical fiber and power splitter have been analyzed. The system has studied according to the number of users, fiber length, coding techniques and fiber dispersion. The transmission of both the network MDM-TDM is experimentally demonstrated over $1.8-\mathrm{km}$ bidirectional optical fiber with bit error-free performance to validate the concept. The result has been observing that when the number of users and fiber length has been increasing, bit error rate is increased as well, but the maximum Q factor and eye height are decreased due to dispersion. As a result of these two most important features, these have an effect on the necessities for future optical mechanisms and technology.

\section{REFERENCES}

[1] J. Li, T. Hu, F. Ren, P. Zhu, Q. Mo, Y. He, Z. Li, and Z. Chen, "Hybrid passive optical network enabled by mode-divisionmultiplexing," 2015 14th International Conference on Optical Communications and Networks (ICOCN), pp. 4673-7373, Sep. 2015.

[2] N. Naim, A. Bakar and M. Ab-Rahman, "Real-time monitoring of passive optical access networks using L-band ASE and varied bandwidth and reflectivity of fiber Bragg gratings", Optics \& Laser Technology, vol. 79, pp. 45-51, 2016.

[3] O. Lopez, C. Daussy, C. Chardonnet, G. Santarelli and A. Amy-Klein, "Frequency dissemination with an 86-km optical fiber for fundamental tests of physics", Ann. Phys. Fr., vol. 32, no. 2-3, pp. 187-189, 2007.

[4] S. D. Ravneet Kaur, A Review on Passive Optical Network, International Journal of Innovative Research in Computer and Communication Engineering, vol. 3(4), 2015.

[5] G. F. M. Lima, E. Lamounier, S. Barcelos, A. Cardoso, I. Peretta, W. Muramoto and F. Barbara, Applying a Differential Evolutionary Algorithm to a Constraint-based System to support Separation of OTDR Superimposed Signal after Passive Optical Network Splitters, CAPES, and FIBERWORK, 2016.

[6] S. Kakkar, Empirical Study of Ethernet Passive Optical Network, IJEAR, pp.2249-4944, Vol 3(1), 2013.

[7] Min Zhu. Research on key techniques in passive optical networks and optical grid applications. Other. 'Ecole Normalesup'erieure de Cachan - ENS Cachan, 2012.

[8] T. Muciaccia, F. Gargano and V. Passaro, "Passive Optical Access Networks: State of the Art and Future Evolution", Photonics, vol. 1, no. 4, pp. 323-346, 2014.

[9] N. Mohd Radzi, "Recent Dynamic Bandwidth Allocation Algorithm Methods In Ethernet Passive Optical Network", IJNCAA, vol. 4, no. 4, pp. 167-176, 2014.

[10] D. M. Ruchi Malhotra, Performance analysis in Passive Optical Networks (PONs), International Journal of Advanced Research in Computer and Communication Engineering, vol.1(4),2012. 
[11] M. Ma, Y. Zhu, and T. Cheng, "A bandwidth guaranteed polling MAC protocol for Ethernet passive optical networks," IEEE INFOCOM 2003. Twenty-second Annual Joint Conference of the IEEE Computer and Communications Societies (IEEE Cat. No.03CH37428), 2003.

[12] K. S., N. Moradpoor, Simulation and Performance Evaluation of Bandwidth Allocation Algorithms for Ethernet Passive Optical Networks (EPONs), University of Ulster, 2015.

[13] A. Shami, Xiaofeng Bai, C. Assi and N. Ghani, "Jitter performance in Ethernet passive optical networks", J. Lightwave Technol., vol. 23, no. 4, pp. 1745-1753, 2005. 